\title{
SKINmages
}

\section{Recognizing Adnexal Tumors in Skin of Color}

Jessica Kay Sterner, BA ${ }^{1}$, Benjamin Rushing, BA², Gage Rensch, MD $^{3}$

${ }^{1}$ University of Missouri School of Medicine

${ }^{2}$ University of Mississippi Medical Center School of Medicine

${ }^{3}$ Tulane University Department of Dermatology

The eccrine poroma is a benign adnexal tumor composed of epithelial cells originating from the terminal duct of the sweat gland. The tumor is rare, with an incidence of 0.001 to $0.008 \%{ }^{1}$ Radiation exposure has been suggested to trigger the development of poromas as case reports have documented multiple poromas in areas of chronic radiation dermatitis. Poromas are not known to have any ethnic, racial, familial or sex predilection. ${ }^{2}$ Patients of any age may be affected, but incidence increases in adulthood. Poromas typically present as an asymptomatic, slow-growing papule, nodule, or plaque. Their color can vary from skincolored, red, brown, or bluish, and typically occur on the palms and soles. ${ }^{3}$

Our patient was a 64-year-old Mexican male presenting to dermatology clinic for a lesion 
on his left abdomen. He reported that the lesion had been slowly enlarging for two years and was asymptomatic. The patient had no personal or family history of skin cancer. Physical examination revealed a pink to grey, polypoid tumor on the left abdomen (Figure 1). The differential diagnosis included acrochordon, condyloma, verrucous carcinoma, basal cell carcinoma, and melanoma. Histopathology showed a polypoid tumor with a markedly acanthotic epidermis with broad interconnecting rete containing small uniform rounded squamous cells. There were foci of vacuoles compatible with poral differentiation and some gland-like lumina. The tumor was diagnosed as an eccrine poroma. There were some pigmented cells and a Melan-A immunostain highlighted some dendritic melanocytes in focal areas within the tumor. This case highlights the importance of recognizing pigmented adnexal tumors in skin of color and represents a rare location for an eccrine poroma.

Conflict of Interest Disclosures: None

Funding: None

Acknowledgements: Andrea Murina, MD

Corresponding Author:

Jessica Sterner, BS

1 Hospital Dr.

Columbia, MO 65212

Phone: 6362190780

Email: jksdm3@health.missouri.edu

\section{References:}

1. Cárdenas ML, Díaz CJ, Rueda R. Pigmented Eccrine Poroma in abdominal region, a rare presentation. Colomb Med (Cali). 2013;44(2):115-117. Published 2013 Jun 30.

2. Ahmed jan N, Masood S. Poroma. [Updated 2021 Jul 20]. In: StatPearls [Internet]. Treasure Island (FL): StatPearls Publishing; 2021 Jan-.
3. Wankhade V, Singh R, Sadhwani V, Kodate P. Eccrine poroma. Indian Dermatol Online J. 2015 Jul-Aug;6(4):304-5. doi: 10.4103/22295178.160296. PMID: 26225348; PMCID: PMC4513423. 